\title{
A Functional Assay for Sick Sinus Syndrome Genetic Variants
}

\author{
Chuanchau J. Jou ${ }^{\mathrm{a}}$ Cammon B. Arrington ${ }^{\mathrm{b}}$ Spencer Barnett ${ }^{\mathrm{b}}$ Jiaxiang Shen ${ }^{\mathrm{c}}$ \\ Scott Cho ${ }^{b}$ Xiaoming Sheng ${ }^{d}$ Patrick C. McCullagh ${ }^{b}$ Neil E. Bowles ${ }^{b}$ \\ Chase M. Pribble $^{b}$ Elizabeth V. Saarel ${ }^{a}$ Thomas A. Pilcher ${ }^{b}$ Susan P. Etheridge ${ }^{b}$ \\ Martin Tristani-Firouzi ${ }^{\text {be }}$ \\ aDivision of Pediatric Cardiology, Cleveland Clinic, Cleveland, 'bivision of Pediatric Cardiology, \\ University of Utah School of Medicine, Salt Lake City, 'Department of Neurobiology and Anatomy, \\ University of Utah, Salt Lake City, dDepartment of Pediatrics, University of Utah, Salt Lake City, eNora \\ Eccles Harrison Cardiovascular Research and Training Institute, University of Utah, Salt Lake City, USA
}

\section{Key Words}

Arrhythmia $\cdot$ Genetics $•$ Sick sinus syndrome $\cdot$ Zebrafish $•$ Sudden cardiac death

\begin{abstract}
Background/Aims: Congenital Sick Sinus Syndrome (SSS) is a disorder associated with sudden cardiac death due to severe bradycardia and prolonged pauses. Mutations in HCN4, the gene encoding inward $\mathrm{Na}^{+} / \mathrm{K}^{+}$current $\left(I_{f}\right)$, have been described as a cause of congenital SSS. The objective of this study is to develop an SSS model in embryonic zebrafish, and use zebrafish as a moderate-throughput assay to functionally characterize $\mathrm{HCN} 4$ variants. Methods: To determine the function of hon4 in zebrafish, embryos were either bathed in the $I_{f}$ -specific blocker (ZD-7288), or endogenous hon4 expression was knocked down using spliceblocking morpholinos. To assess whether the zebrafish model discriminates benign from pathogenic variants, we tested four HCN4 mutations known to cause human SSS and four variants of unknown significance (VUS). Results: Pharmacological blockade and knockdown of hon4 in zebrafish phenocopied human SSS, displaying bradycardia and cardiac pauses in intact embryos and explanted hearts. The zebrafish assay correctly identified all diseasecausing variants. Of the VUS, the assay predicted 2 as benign and 2 as hypomorphic variants. Conclusions: We conclude that our embryonic zebrafish assay is a novel and effective tool to functionally characterize human HCN4 variants, which can be translated into important clinical prognostic information.
\end{abstract}

C. J. Jou and C. B. Arrington contributed equally to this work.

Martin Tristani-Firouzi, MD and Chuanchau J. Jou

KARGER
Department of Pediatrics (Cardiology), University of Utah School of Medicine, 81 N Mario Capecchi Drive, Salt Lake City, Utah 84113, (USA); Department of Pediatrics (Cardiology), Cleveland Clinic, 9500 Euclid Ave., Cleveland, $\mathrm{OH}$, (USA)

Tel. 801-213-7606, E-Mail Martin.Tristani@utah.edu / jerry_jou@hotmail.com 


\section{Introduction}

The sinoatrial node, originally described by Keith and Flack in 1907, is the cardiac pacemaker that generates and maintains cardiac automaticity. Sinoatrial node dysfunction or Sick Sinus Syndrome (SSS) causes bradyarrhythmia with a broad clinical spectrum ranging from no symptoms to sudden cardiac death [1]. Mutations in HCN4 have been shown to cause sporadic and familial SSS $[1,2]$. HCN4 encodes the potassium/sodium hyperpolarization-activated cyclic nucleotide-gated channel 4 , which contributes to the native pacemaker currents in the heart $\left(\mathrm{I}_{\mathrm{f}}\right)$. Traditionally the functionality of $\mathrm{HCN} 4$ variants has been determined by assays such as heterologous expression systems or non-human myocytes [2]. Unfortunately these assays are too laborious to functionally characterize the many variants currently detected by [1] genetic testing. Thus, the majority of $H C N 4$ variants are without functional interpretation and classified as variants of unknown significance (VUS) [3].

Hcn 4 knock-out mice have been extensively studied [4, 5]. However, the utility of the mouse knock-out model has been limited due to embryonic lethality in homozygous knockout mice and the absence of a phenotype in heterozygous mice [4]. Zebrafish display many similar cardiac electrophysiological characteristics as humans and are more amenable to high-throughput functional experiments [6]. We have previously developed an in vivo zebrafish assay to characterize the functional consequences of human Long QT Syndrome genetic variants [7]. We now present another in vivo cardiac assay to functionally characterize potential SSS genetic variants with the goal to provide meaningful clinical interpretation to HCN4 variants detected by genetic testing. In this study, we show that knockdown of hcn 4 in zebrafish phenocopies human SSS. Furthermore, we show that the zebrafish assay can effectively discriminate between deleterious and benign human HCN4 variants.

\section{Materials and Methods}

Human and animal subject research for this study was approved by the University of Utah Institutional Review Board. The human subjects gave informed consent.

\section{Zebrafish strains and characterization of the cardiac phenotype}

Wild-type (WT) or Tg (cmlc2:GFP) zebrafish (Danio rerio) embryos were used to functionally characterize zebrafish $h c n 4$ and human $H C N 4$ variants. Embryos were maintained in embryo water at $28^{\circ} \mathrm{C}$ and staged according to age and morphological criteria. Cardiac phenotypes were screened using light (WT embryos) or fluorescent (Tg cmlc2:GFP) microscopy at 36-40 hour-post-fertilization (hpf). Heart rate and rhythm were recorded. Videos obtained from the Tg embryos were analyzed using Image J to determine the frequency and duration of cardiac pauses.

\section{Molecular biology}

Human HCN4 cDNA (original expression vector provided by Michael Sanguinetti) was subcloned into the pCS2+ expression vector. The QuikChange site-directed mutagenesis kit (Agilent Technologies) was used to introduce mutations into WT HCN 4 cDNA. The presence of mutations and absence of mutagenesis artifacts were confirmed by Sanger sequencing of selected clones at the University of Utah DNA sequencing core. Plasmids were linearized with HpaI, and cRNA was prepared using the mMessage mMachine T7 in vitro transcription kit (Ambion) and purified with Qiagen RNeasy kit. cRNA concentrations were measured using the NanoDrop RNA quantitation kit.

Pharmacological blockade of hon 4

Intact zebrafish embryos

ZD-7288 (Sigma) was dissolved in distilled water (stock, $10 \mathrm{mM}$ ). After fertilization, WT embryos were allowed to develop in standard embryo water at $28^{\circ} \mathrm{C}$. At $24 \mathrm{hpf}$, embryos were transferred to $1 \mathrm{mM} \mathrm{ZD}$ 7288 solution in embryo water and incubated at $28^{\circ} \mathrm{C}$ for 24 hours. The heart rate of non-treated (control) and ZD-7288 treated embryos was recorded at $48 \mathrm{hpf}$. 
Explanted zebrafish hearts

ZD-7288 was dissolved in external control solution containing (in mmol/L) $\mathrm{NaCl} 140, \mathrm{KCl} 4, \mathrm{CaCl}_{2}$ 1.8, $\mathrm{MgCl}_{2} 1$, glucose 10, and HEPES $10(\mathrm{pH} 7.4)$ to a concentration of $10 \mathrm{uM}$. Hearts from WT embryos (48 hpf) were dissected from the thorax en bloc by using fine forceps and transferred to the recording chamber. Only spontaneously beating whole hearts were studied. All experiments were performed at $22^{\circ} \mathrm{C}$ to $24^{\circ} \mathrm{C}$. The recording chamber was superfused with external control solution or ZD-7288 solution. Suction pipettes were made from borosilicate capillary tubes ( 8250 glass; A-M Systems) and fire-polished to obtain resistances of 6-9 $\mathrm{M} \Omega$ when filled with solution containing (in mmol/L) KCl 120, EGTA 5, $\mathrm{K}_{2} \mathrm{ATP} 5, \mathrm{MgCl}_{2} 5$, and HEPES 10 ( $\mathrm{pH} 7.2)$.

$V_{\mathrm{m}}$ was measured by using an AxoClamp 2A amplifier (Molecular Devices) in the bridge mode with the disrupted patch technique. The pipette was positioned adjacent to the heart and a seal was formed by application of minimal suction. Using this technique, stable spontaneous action potentials (APs) were recordable for up to 3 hours. $V_{\mathrm{m}}$ was filtered at $10 \mathrm{kHz}$ and digitized at a sampling frequency of $20 \mathrm{kHz}$ with a 12-bit analog-to-digital converter (Digidata 1322A Interface, Molecular Devices).

Injection of morpholinos and synthesized mRNA

Hcn4 splice-blocking morpholino (SB-MO) was designed to target the exon3-intron3 boundary of hcn 4 (5'-TTTAGACACTCACCACCATCTTGTT-3'). Hcn4 SB-MO was injected (10 ng/embryo) alone or co-injected with wild-type (WT) or mutant $H C N 4$ cRNAs (300 pg/embryo) at the 1- to 2-cell stage. All SB-MO / cRNA injections were repeated at least three times.

In situ hybridization

A digoxigenin-labeled antisense RNA probe was synthesized using T3 polymerase by in vitro transcription of a cDNA template to $h c n 4$. Whole mount in situ hybridization was performed using a Biolane HTI in situ machine (Huller and Huttner AG). Zebrafish embryos were cleared in 70\% glycerol in Phosphate Buffered Saline Tween. Embryos were photographed with a Leica MZ12 stereo microscope using a DageMTI DC330 CCD camera.

\section{HCN4 variant screening}

DNA was isolated from the subject's peripheral blood using a Gentra Autopure LS (Qiagen, Valencia, CA) and analyzed by agarose gel electrophoresis to confirm the integrity of the DNA and quantitated using a Nanodrop. PCR primers (Table 5) were designed to amplify the exons of HCN4 using the ExonPrimer utility in the UCSC genome browser (http://genome.ucsc.edu/), and used to amplify DNA from the patient. An aliquot of DNA was analyzed by agarose gel electrophoresis and then the PCR product was purified by treating with $4 \mu$ of Exo-SAP-IT (Affymetrix) at 37 C for 2 hours and $80^{\circ} \mathrm{C}$ for 15 minutes. The PCR product was then submitted to the University of Utah DNA sequencing core for analysis and results compared to published sequences using BLAST (http://blast.ncbi. nlm.nih.gov/Blast.cgi). To predict deleterious effects, the genetic variants were analyzed using the algorithms SIFT [8], PolyPhen2 [9], MutationTaster [10], PROVEAN [11], FATHMM [12] and MutationAsssesor [13].

\section{Statistical analysis}

pCLAMP 10 software (Molecular Devices) was used to analyze digitized data. All data are expressed as mean \pm SEM ( $n=$ number of sample), and statistical significance was evaluated by a Student's $t$ - test or Analysis of Variance (ANOVA) where appropriate ( $\mathrm{P} \leq 0.05$ was considered a statistically significant difference). Atrial and ventricular AP parameters, including maximum upstroke velocity $\left(\mathrm{P}_{\text {max }}\right.$ ), action potential duration (APD), maximum diastolic membrane potential (MDP), and cycle length (CL) were independently analyzed. Action potential duration from a series of five APs was calculated as the time interval between the peak maximum upstroke velocity (phase 0 ) and the time at $10 \%\left(\mathrm{APD}_{10}\right), 50 \%\left(\mathrm{APD}_{50}\right)$ and $90 \%$ of repolarization $\left(\mathrm{APD}_{90}\right)$. Values are given as mean $\pm \mathrm{SEM}$.

A Generalized Linear Mixed Model was performed to estimate the likelihood of a normal phenotype for the HCN4 variant, compared to the reference normal phenotype for WT HCN4. A random intercept was specified in the Generalized Linear Mixed Model to account for day to day experimental variability. The response variable, cardiac pause was a binary outcome dichotomized as normal (no pause) and abnormal (with pause) phenotypes. The response variable, heart rate, was a continuous variable. The percentage of 


\section{Cellular Physiology Cell Physiol Biochem 2017;42:2021-2029 \begin{tabular}{ll|l} 
and Biochemistry Published online: August 11, 2017 & $\begin{array}{l}\text { (C) } 2017 \text { The Author(s). Published by S. Karger AG, Basel } \\
\text { www.karger.com/cpb }\end{array}$ \\
\hline
\end{tabular}

embryos with a normal phenotype and the 95\% confidence interval were calculated for each HCN4 mutant using the binominal proportion method with the normal approximation assumption.

\section{Results}

\section{Drug inhibition of hon 4 in zebrafish}

The zebrafish gene hcn4 (XP_686078) encodes a protein of 1130 amino acids that by alignment appears to be the orthologue of human HCN4. To explore whether hcn4 controls heart rate and cardiac automaticity in zebrafish, we analyzed the spatial and temporal distribution of hcn 4 expression during zebrafish development. We found that hcn 4 is strongly expressed in the zebrafish heart after formation of the linear heart tube (Fig. 1). Given these expression and protein alignment data we reasoned that $h c n 4$ is the functional orthologue of $\mathrm{HCN} 4$.

To explore the mechanism by which hon 4 functions in zebrafish, we bathed whole embryos in 4-(N-ethyl-N-phenylamino)-1,2-dimethyl-6-(methylamino) pyrimidinium chloride (ZD-7288), a nonselective hcn blocker, and assayed the heart rate at $48 \mathrm{hpf}$. We found that embryos bathed in $1 \mathrm{mM} \mathrm{ZD-7288}$ had significantly slower heart rates than untreated embryos (Fig. 2A). However, cardiac pauses were not observed in embryos exposed to ZD7288.

To further characterize the drug-induced bradycardia, explanted zebrafish hearts were superfused with ZD-7288 (10 uM) and patch-clamped to measure cardiac AP parameters. As in the intact embryos, CL was significantly prolonged in both the atrium and ventricle after the drug treatment (Table 1). Rate-dependent $\mathrm{APD}$ prolongation $\left(\mathrm{APD}_{50}\right.$ and $\left.\mathrm{APD}_{90}\right)$

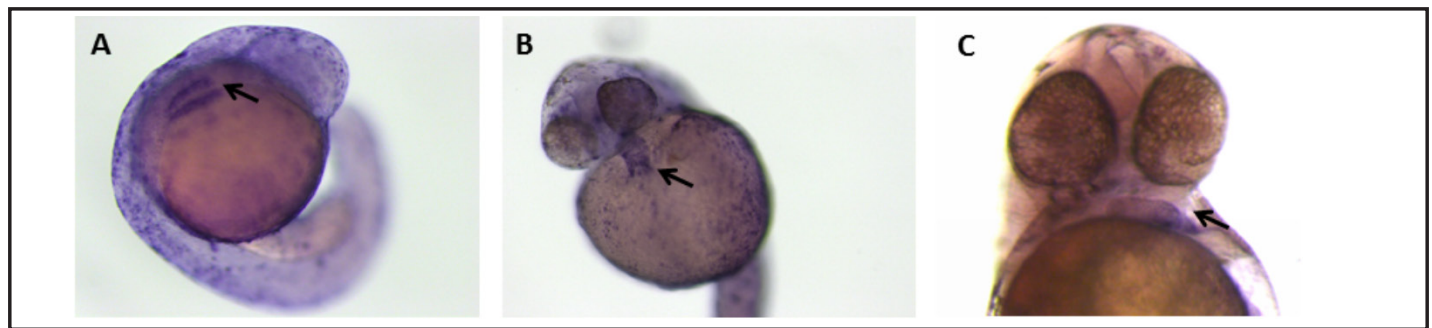

Fig. 1. Embryonic hnc4 expression: whole-mount in situ hybridization reveals hcn4 expression in the heart (arrow) at (A) 18 somites, (B) $24 \mathrm{hpf}$ and (C) $48 \mathrm{hpf}$.
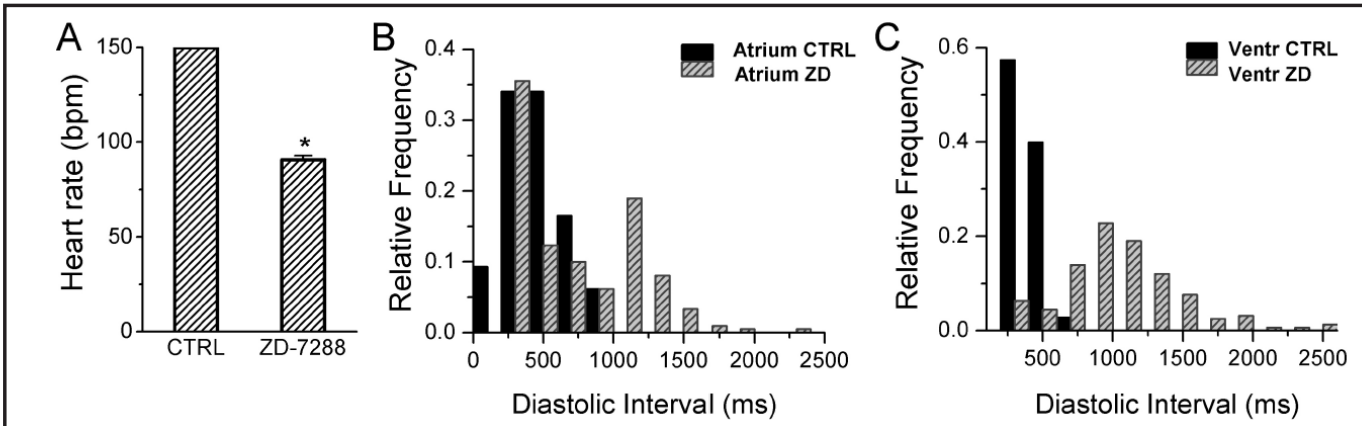

Fig. 2. Effects of pharmacological blockade of hen channels on in vivo and ex vivo cardiac activity. (A) The nonselective hcn blocker ZD-7288 slows heart rate in intact embryonic zebrafish. Embryos were bathed with 1 mM ZD-7288 ( $\mathrm{n}=10$ paired experiments). (B and C) Histograms of diastolic intervals obtained from current clamp recordings of explanted embryonic hearts before (CTRL) and after perfusion with $10 \mu \mathrm{M}$ ZD-7288 (ZD). The diastolic interval is a surrogate for the rate of phase 4 diastolic depolarization. For both atrial and ventricular recordings, ZD prolonged the diastolic intervals, consistent with slowing of phase 4 depolarization rate. 
Fig. 3. Effects of hcn4 MO-knockdown on heart rate and cardiac pauses. (A) hcn 4 MO-knockdown slowed sinus heart rate in intact embryos at 3648 hpf (n = 209), *p < 0.0001. (B) hcn4 MO-injected embryos displayed sinus pauses that were never detected in control embryos. (C) Continuous recordings of cardiac contraction in $\mathrm{cmcl2}$-GFP control embryos (left) and embyros injected with hon4 MO. Embyros injected with hon4 MO displayed bradycardia and prolonged pauses, reminiscent of patients with SSS.

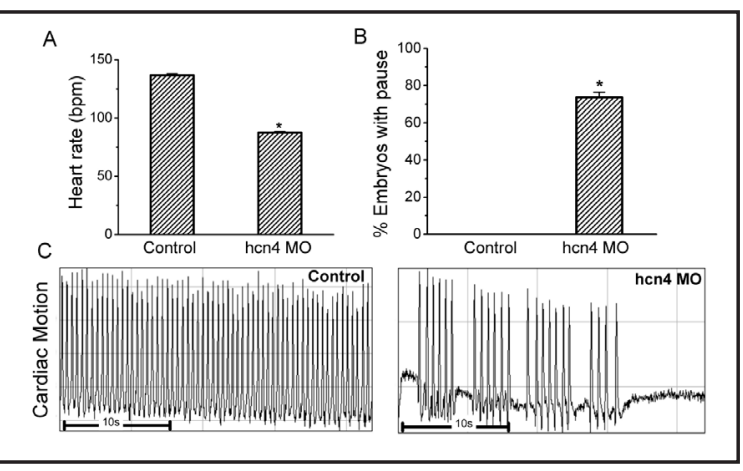

was observed in the ventricles after ZD7288 treatment, but the rate-dependent phenomenon was not present in the atrium. Unlike the intact embryos, cardiac pauses were observed in the explanted zebrafish heart superfused with ZD-7288.

Diastolic intervals were tabulated from the patch-clamp recordings. Treatment with ZD-7288 resulted in prolongation of diastolic intervals in both the atrium and ventricle as demonstrated by the histograms in Fig. 2B and $2 \mathrm{C}$. The atrial diastolic intervals were $441 \pm 20 \mathrm{~ms}$ and $1033 \pm 130 \mathrm{~ms}$ for control and ZD treatment, respectively (mean \pm SEM; $\mathrm{N}=97$ and 211, respectively; $\mathrm{p}<0.005$; ANOVA). Ventricular diastolic intervals were $366 \pm 11 \mathrm{~ms}$ and $1387 \pm 142 \mathrm{~ms}$ for control and ZD treatment, respectively $(\mathrm{N}=143$ and 158, respectively; $\mathrm{p}<0.005$; ANOVA). Taking the diastolic interval as a surrogate for phase 4 diastolic depolarization, the AP phase known to be controlled by $H C N 4$, these results support the notion that a member of the hcn family of ion channels plays a role in controlling zebrafish heart rate.

Morpholino hon4 knock-down produces bradyarrhythmias

To determine whether hon 4 is one of the ion channels responsible for heart rate control in zebrafish, we performed targeted hon4 knock-down experiments with splice-blocking antisense morpholino oligonucleotide. The overall appearance of hon 4 knock-down morphants was normal (data not shown). Zebrafish cardiac phenotypes, including heart rate and cardiac rhythm, were assessed between 36 and 40 hpf. The phenotype observed in zebrafish hcn 4 morphants was strikingly similar to that observed in humans SSS, and included bradycardia and prolonged sinus pauses,

Table 1. Atrial (A) and ventricular (B) action potential parameters in response to ZD-7288, measured in explanted embryonic zebrafish heart. CL: cycle length (msec); MDP: maximum diastolic potential (mV); Pmax: maximum upstroke velocity $(\mathrm{mV} /$ msec); AP10, AP50 or AP90: action potential duration to $10 \%, 50 \%$ or $90 \%$ of repolarization (msec). Values are mean \pm SEM

\begin{tabular}{|c|c|c|c|}
\hline $\begin{array}{c}\text { A } \\
\text { Atrium }\end{array}$ & $\begin{array}{l}\text { Uninjected } \\
(n=4)\end{array}$ & $\begin{array}{l}\text { Hcn4 morph. } \\
(n=4)\end{array}$ & $\mathrm{P}$-value \\
\hline CL & $723.3 \pm 53.7$ & $1845 \pm 443.3$ & 0.04 \\
\hline MDP & $-63.4 \pm 1.9$ & $-62.2 \pm 2.4$ & 0.80 \\
\hline Pmax & $4.6 \pm 0.4$ & $6.8 \pm 0.8$ & 0.07 \\
\hline $\mathrm{APD}_{10}$ & $57.7 \pm 4.2$ & $60.6 \pm 11.5$ & 0.82 \\
\hline $\mathrm{APD}_{50}$ & $103.2 \pm 6.8$ & $110.5 \pm 15.6$ & 0.68 \\
\hline $\mathrm{APD}_{90}$ & $135.1 \pm 6.3$ & $156.5 \pm 14.5$ & 0.22 \\
\hline $\begin{array}{c}\text { B } \\
\text { Ventricle }\end{array}$ & $\begin{array}{l}\text { Uninjected } \\
(n=4)\end{array}$ & $\begin{array}{l}\text { Hcn4 morph. } \\
(n=4)\end{array}$ & P-value \\
\hline $\mathrm{CL}$ & $877.4 \pm 53.2$ & $1291 \pm 154.6$ & 0.04 \\
\hline MDP & $-65.6 \pm 2.1$ & $-66.5 \pm 1.8$ & 0.75 \\
\hline Pmax & $6.7 \pm 0.6$ & $5.2 \pm 1.5$ & 0.39 \\
\hline $\mathrm{APD}_{10}$ & $207.3 \pm 20.8$ & $232.4 \pm 28.8$ & 0.50 \\
\hline $\mathrm{APD}_{50}$ & $359.2 \pm 23.4$ & $383.8 \pm 33.6$ & 0.57 \\
\hline APD90 & $401.1 \pm 24.4$ & $429.4 \pm 30.8$ & 0.49 \\
\hline
\end{tabular}

Table 2. Atrial (A) and ventricular (B) action potential parameters of uninjected and hcn 4 morphant explanted embryonic heart

\begin{tabular}{cccc}
\hline A & & & \\
Atrium & Control $(n=5)$ & ZD-7288 $(n=5)$ & P-value \\
\hline CL & $724.6 \pm 115.6$ & $1481 \pm 248.1$ & $<0.01$ \\
MDP & $-71.0 \pm 2.5$ & $-70.7 \pm 3.5$ & 0.85 \\
Pmax & $11.9 \pm 3.0$ & $7.5 \pm 1.6$ & 0.06 \\
APD $_{10}$ & $60.8 \pm 9.3$ & $61.5 \pm 5.9$ & 0.89 \\
APD $_{50}$ & $101.5 \pm 13.0$ & $109.1 \pm 12.7$ & 0.37 \\
APD 90 & $127.2 \pm 17.4$ & $150.8 \pm 15.1$ & 0.14 \\
\hline B & & & \\
Ventricle & Control $(n=5)$ & ZD-7288 (n=5) & P-value \\
\hline CL & $786.2 \pm 115.9$ & $1504 \pm 163.9$ & 0.01 \\
MDP & $-69.8 \pm 0.9$ & $-70.3 \pm 1.1$ & 0.53 \\
Pmax & $5.2 \pm 4.1$ & $4.6 \pm 2.9$ & 0.30 \\
APD 10 & $256.6 \pm 30.2$ & $298.6 \pm 36.3$ & 0.02 \\
APD & $532.6 \pm 60.2$ & $624.2 \pm 66.4$ & $<0.01$ \\
APD 90 & $598.2 \pm 115.9$ & $713.6 \pm 75.7$ & $<0.01$ \\
\hline
\end{tabular}




\section{Cellular Physiology Cell Physiol Biochem 2017;42:2021-2029

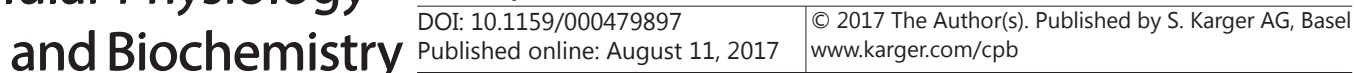 Jou et al.: Hen4 Assays for Sick Sinus Syndrome}

Fig. 4. Specificity of hcn 4 MO. Retention of intron 3 with injection of hcn 4 splice-blocking morpholino. RNA from uninjected and morphant embryos was analyzed by RT-PCR. Primers designed to amplify the exon3-exon4 boundary produced the expected PCR product in uninjected embryos (lane 2). The PCR product is too large to amplify in morphants due to inclusion of intron 3 (lane 3 ). To prove inclusion of intron 3 in morphant hcn 4 mRNA, primers were designed to amplify the exon3-intron3 and intron3-exon 4 boundaries. Products of the expected size were found in morphants (lanes 4 and 5, respectively).

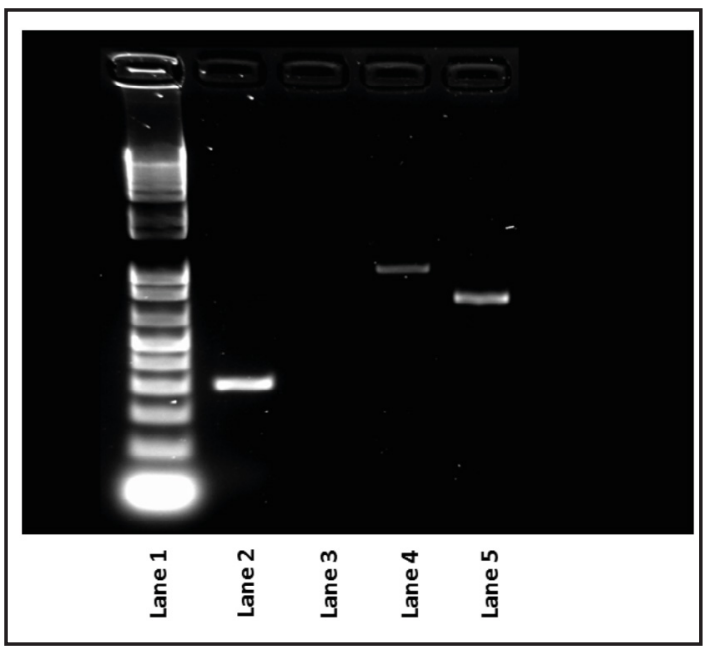

the latter of which are thought to be the cause of syncope and sudden death in SSS. The morphant heart rate $(n=209)$ was significantly slower than in uninjected $(n=57)$ embryos (88 vs 137 beats/minute; Fig. 3A). In addition, there were intermittent periods of asystole (pauses) lasting several seconds in 74\% of morphants (Fig. 3B and 3C). Such pauses were never observed in uninjected embryos. In the morphants, there were $3.6 \pm 0.3$ pauses/ minute and the average pause duration was $2.3 \pm 0.4$ seconds.

Current-clamp experiments of explanted hearts revealed a significant difference in CL between morphants and uninjected embryos, but none of the other AP parameters were significantly different. Unlike the pharmacological blockade of $I_{f}$ by ZD-7288, the ratedependent APD prolongation was not observed in morphant embryos (Table 2).

Evidence supporting morpholino specificity comes from RT-PCR analysis of hcn4 splice-blocking MO injected embryos, in which there was retention of hcn 4 intron 3, which is predicted to cause premature translation termination (Fig. 4). As additional evidence for morpholino specificity, the heart rate and pause phenotypes were partially rescued (from 87 to $111 \mathrm{bpm}$ and from $74 \%$ to $25 \%$, respectively) with co-injection of WT human HCN4 mRNA, suggesting that HCN4 can compensate for the loss of the zebrafish orthologue (Fig. 5).

\section{High-throughput HCN4 variant screen}

Next, we tested $H C N 4$ variants using the zebrafish assay to determine the functional consequences. We selected HCN4 variants (p.P174S, p.G480R, p.A485V, p.D553N) that have been identified in patients diagnosed with congenital SSS $(8,9,10,11)$. The p.P174S variant is a novel mutation identified in a patient from our clinic with bradycardia and resuscitated sudden cardiac death (Table 3). Compared to the WT rescue of heart rate and pause, the degree of rescue by all four putative disease-causing variants were statistically lower than the WT (Fig. 5). For example, there was no statistical difference between pause frequency or pause duration between morphants alone ( $3.6 \pm 0.3$ pauses/minute and $2.3 \pm 0.4$ seconds) and morphants co-injected with the HCN4 variant p.P174S ( $4.8 \pm 0.9$ pauses/minute and 3.9 \pm 0.7 seconds), suggesting that the p.P174S variant is indeed non-functional and diseasecausing.

In addition to the pathogenic variants, we also tested HCN4 variants of unknown significance that were identified through the NHLBI Exome Sequencing Project (ESP) database (Table 4). Ideally we would have liked to test $H C N 4$ variants with a high probability of being non-pathogenic by being common (MAF > 0.01) in "unaffected" individuals and predicted benign by in silico programs. However, there are few common non-synonymous variants within the ESP database. Therefore, we decided to focus on variants predicted to be benign by at least 3 of 6 algorithms (SIFT [8], PolyPhen2 [9], MutationTaster [10], PROVEAN [11], FATHMM [12] and MutationAsssesor [13]): p.G36E (rs143090627: MAF 0.05 in European Americans), p.T766A (rs372090359: MAF 0.0001), p.R934H (rs369485237: MAF 0.0001), 


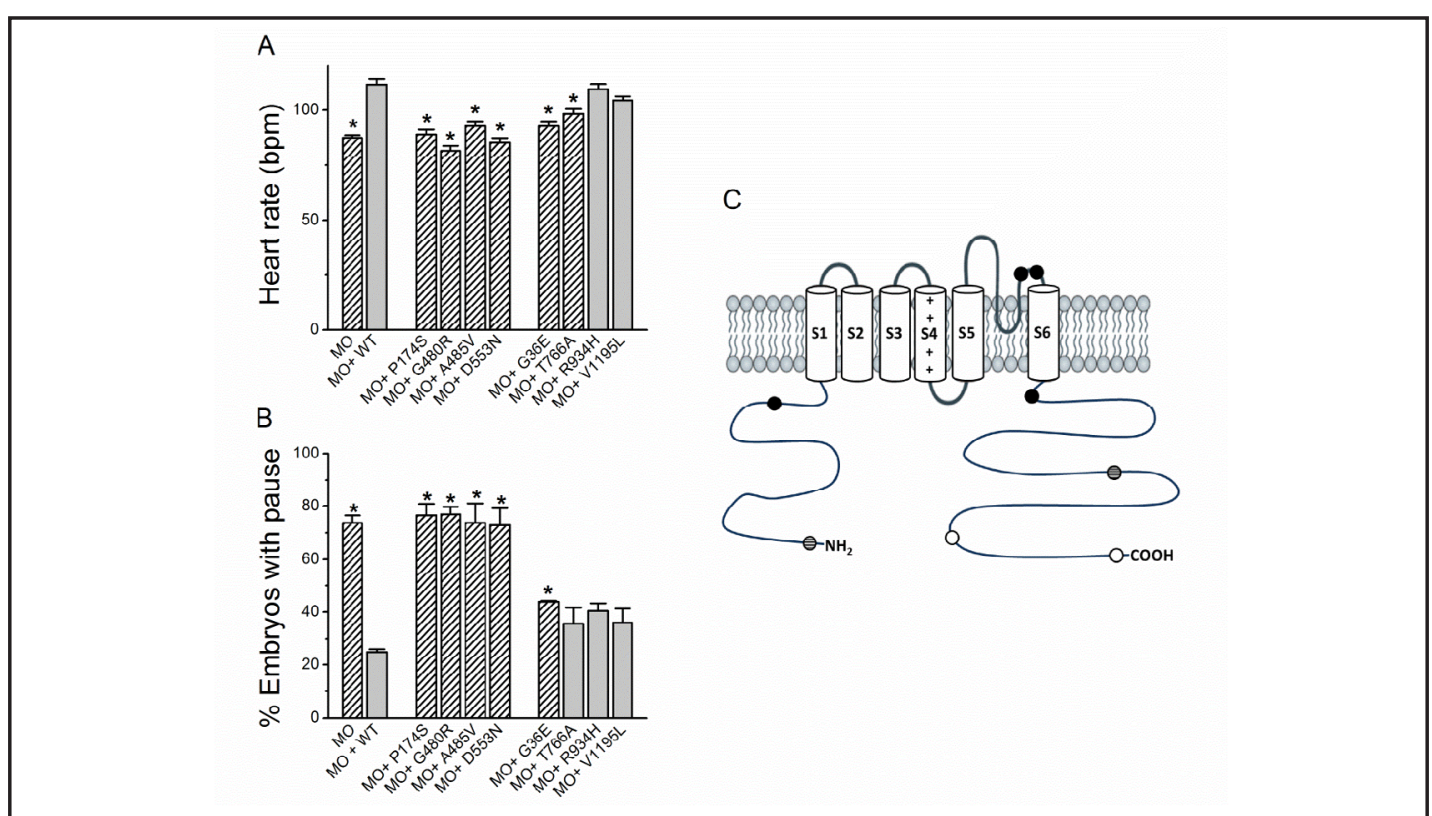

Fig. 5. An in vivo cardiac assay to distinguish benign and pathogenic HCN4 variants. The effects of MO-knockdown and the degree of rescue by co-injection of human WT or mutant HCN4 are displayed for heart rate (A) and sinus pauses (B). Fertilized embryos were injected with hcn $4 \mathrm{MO}$ alone or in combination with WT or mutant HCN4 at the 1-2 cell stage and assayed for heart rate and pauses at 36-40 hpf. R934H (n = 57) and V1195L ( $\mathrm{n}=75$ ) variants rescued both heart rate and pauses to a similar degree as WT HCN4, implying that these are benign variants. G36E $(n=66)$ did not rescue heart rate or pauses to the same level as WT; T766A $(n=59)$ rescued pauses, but did not fully rescue the heart rate to the same degree as WT, suggesting that G36E and T766A may function as a hypomorphic alleles. ${ }^{*} \mathrm{P}<0.05$ compared to WT HCN4 co-injection. (C) A schematic diagram of HCN4 subunit showing position of the pathogenic (O), benign $(\bigcirc)$ and intermediate (O) variants.

Table 3. Genotype and phenotype correlation of putative disease-causing HCN4 variants. SCD: sudden cardiac death

\begin{tabular}{cccc}
\hline HCN4 variant & Occurrence & $\begin{array}{c}\text { Average } \\
\text { heart rate }\end{array}$ & Symptom \\
\hline p.P174S & Individual & $51 \mathrm{bpm}$ & Syncope, Resuscitated SCD \\
p.G480R & Single Family & $48 \mathrm{bpm}$ & None \\
p.A485V & Three Families & $58 \mathrm{bpm}$ & Pre-syncope, Resuscitated SCD \\
p.D553N & Single Family, one member indexed & $39 \mathrm{bpm}$ & Resuscitated SCD \\
\hline
\end{tabular}

and p.V1195L (rs143431889: MAF 0.0002 in African Americans). Furthermore none of these variants are found within a known functional domain. The p.R934H and p.V1195L variants rescued the bradycardia and pause phenotypes to a similar level as WT HCN4, suggesting they are functional, benign variants. In contrast, p.G36E only partially rescued the bradycardia and pause phenotypes, while p.T766A rescued the pause phenotype but not the heart rate phenotype. There was no clear relationship between in silico predictions of pathogenicity and the functional rescue for the variants of unknown significance.

\section{Discussion}

Congenital SSS is a heritable disease that is associated with sudden cardiac death. HCN channels that encode $I_{f}$ are a major determinant of diastolic depolarization in sinus node cells and play a primary role controlling cardiac automaticity. There are currently more 
Table 4. Predicted consequences of HCN4 genetic variants using in silico prediction tools

\begin{tabular}{|c|c|c|c|c|c|c|c|c|}
\hline $\begin{array}{c}\text { Genomic } \\
\text { coordinates }\end{array}$ & Consequence & $\begin{array}{l}\text { Allele frequency } \\
\text { (ExaC) }\end{array}$ & SIFT & Polyphen2 & $\begin{array}{l}\text { Mutation } \\
\text { Taster }\end{array}$ & PROVEAN & FATHНM & $\begin{array}{l}\text { Mutation } \\
\text { Assessor }\end{array}$ \\
\hline $15: 73660092 \mathrm{G} / \mathrm{A}$ & p.Pro174Ser & 0,0001276 & Tolerated & Benign & Polymorphism & Neutral & Damaging & Neutral \\
\hline $15: 73622066 \mathrm{C} / \mathrm{G}$ & p.Gly480Arg & novel & Damaging & Probably Damaging & Disease Causing & Deleterious & Damaging & High \\
\hline $15: 73622050 \mathrm{G} / \mathrm{A}$ & p.Ala485Val & novel & Damaging & Probably Damaging & Disease Causing & Deleterious & Damaging & Low \\
\hline $15: 73617719 \mathrm{C} / \mathrm{T}$ & p.Asp553Asn & 0,00002472 & Tolerated & Probably Damaging & Disease Causing & Deleterious & Damaging & Low \\
\hline $15: 73660505 \mathrm{C} / \mathrm{T}$ & p.Gly36Glu & 0,06131 & Damaging & Benign & Polymorphism & Neutral & Damaging & Neutral \\
\hline $15: 73616138 \mathrm{~T} / \mathrm{C}$ & p.Thr766Ala & 0,000008413 & Tolerated & Benign & Polymorphism & Neutral & Tolerated & Neutral \\
\hline $15: 73615633 \mathrm{C} / \mathrm{T}$ & p.Arg934His & 0,00005591 & Tolerated & Benign & Polymorphism & Neutral & Damaging & Neutral \\
\hline $15: 73614851 \mathrm{C} / \mathrm{A}$ & p.Val1195Leu & 0,00001295 & Damaging & Benign & Disease Causing & Neutral & Damaging & Neutral \\
\hline
\end{tabular}

than 150 HCN4 variants listed in the ESP database. Nearly all of the variants have yet to be characterized functionally. In this study, we show that pharmacological and genetic perturbation of hon 4 channels in embryonic zebrafish phenocopied human SSS. In addition to bradycardia, pharmacological blockade of $\mathrm{I}_{\mathrm{f}}$ and genetic knockdown of hcn 4 resulted in prolonged cardiac pauses.

Our results demonstrate that zebrafish can be used as a novel in vivo model to assay the pathogenicity of human HCN4 variants. By assessing both heart rate and cardiac pauses, all four "pathogenic" variants (p.P174S, p.A480R, p.A485V and p.D553N) were accurately identified as disease-causing. Interestingly, the p.G480R variant corresponds to a family of asymptomatic young patients with extreme bradycardia (Table 3), and given the zebrafish findings, perhaps these individuals are susceptible to periods of asystole when they are older.

In contrast to the pathogenic variants, two of the VUS (p.R934H and p.V1195L) behave as WT HCN4. However, the two VUS, p.G36E and p.T766A, exhibit an intermediate effect on heart rate and pause and, based on the degree of rescue, appear to be functionally hypomorphic. Patients with SSS caused by HCN4 mutations are often bradycardic at rest, and range in clinical presentation from asymptomatic to sudden cardiac death. Since the unknown variants we tested herein are from individuals who are probably "normal" or asymptomatic, the hypomorphic HCN4 variants might have been identified in individuals with a subclinical phenotype that is commonly referred to as "asymptomatic bradycardia."

There are several inherent limitations to the assay we developed. First, despite being an in vivo cardiac system, some would argue that zebrafish is not an ideal model for human arrhythmogenic disease since it is a non-mammalian organism with a 2-chamber heart. However, the electrophysiological properties of zebrafish myocardium are more similar to human myocardium than other mammalian systems, such as mice $[7,14]$. Second, while the assay is not particularly quantitative, it does provide a moderate-throughput approach to characterize whether an HCN4 variant is pathogenic, benign or hypomorphic. Third, although it would be ideal to measure $\mathrm{I}_{\mathrm{f}}$ in isolated embryonic zebrafish cardiomyocytes, this experiment is technically challenging because embryonic myocytes cannot survive enzymatic dispersion. We contend that the combination of pharmacological hen 4 blockade and hen 4 morpholino knock-down experiments is a reasonable surrogate method to assay the effect of $I_{f}$ perturbation. Finally, although the assay does not provide a detailed mechanism to explain disease susceptibility, it offers a relatively efficient, cost-effective approach to obtain a functional read-out of $H C N 4$ variants. 
In the current era of medicine, integration of genetic testing into clinical practice has become the norm. However, genetic information without meaningful functional data is a major obstacle that hinders advances in science and medicine. Therefore, our cost-effective and labor-efficient tools such as this HCN4 zebrafish assay should be considered, not only as a research tool, but also as a tool to bridge the gap between gene discovery and clinical practice.

\section{Acknowledgements}

We are indebted to Michael Sanguinetti for providing the wild-type HCN4 plasmid construct. Special thanks to Lisa Mills and Lindsay Myers for their assistance in patient recruitment. This work was supported by Fellow-to Faculty Award from American Heart Association (C.J.J.) and the Nora Eccles Treadwell Foundation (M.T-F.)

\section{Disclosure Statement}

None.

\section{References}

1 Milanesi R, Baruscotti M, Gnecchi-Ruscone T, DiFrancesco D: Familial sinus bradycardia associated with a mutation in the cardiac pacemaker channel. N Engl J Med 2006;354:151-157.

$>2$ Verkerk AO, Wilders R: Pacemaker activity of the human sinoatrial node: effects of HCN4 mutations on the hyperpolarization-activated current. Europace 2014;16:384-395.

- 3 Eggington JM, Bowles KR, Moyes K, Manley S, Esterling L, Sizemore S, Rosenthal E, Theisen A, Saam J, Arnell C, Pruss D, Bennett J, Burbidge LA, Roa B, Wenstrup RJ: A comprehensive laboratory-based program for classification of variants of uncertain significance in hereditary cancer genes. Clin Genet 2014;86:229-237.

4 Stieber J, Herrmann S, Feil S, Löster J, Feil R, Biel M, Hofmann F, Ludwig A: The hyperpolarization-activated channel HCN4 is required for the generation of pacemaker action potentials in the embryonic heart. Proc Natl Acad Sci USA 2003;100:15235-15240.

5 Baruscotti M, Bucchi A, Viscomi C, Mandelli G, Consalez G, Gnecchi-Rusconi T, Montano N, Casali KR, Micheloni S, Barbuti A, DiFrancesco D: Deep bradycardia and heart block caused by inducible cardiacspecific knockout of the pacemaker channel gene Hcn4. Proc Natl Acad Sci USA 2011;108:1705-1710.

6 Peal DS, Peterson RT, Milan D: Small molecule screening in zebrafish. J Cardiovasc Transl Res 2010;3:454460.

7 Jou CJ, Barnett SM, Bian JT, Weng HC, Sheng X, Tristani-Firouzi M: An in vivo cardiac assay to determine the functional consequences of putative long QT syndrome mutations. Circ Res 2013;112:826-830.

8 Ng PC, Henikoff S: SIFT: Predicting amino acid changes that affect protein function. Nucleic Acids Res 2003;31:3812-3814.

-9 Adzhubei I, Jordan DM, Sunyaev SR: Predicting functional effect of human missense mutations using PolyPhen-2. Curr Protoc Hum Genet 2013; Chapter 7:Unit7 20.

10 Schwarz JM, Rodelsperger C, Schuelke M, Seelow D: MutationTaster evaluates disease-causing potential of sequence alterations. Nat Methods 2010;7:575-576.

11 Choi Y, Sims GE, Murphy S, Miller JR, Chan AP: Predicting the functional effect of amino acid substitutions and indels. PLoS One 2012;7:e46688.

12 Shihab HA, Gough J, Cooper DN, Stenson PD, Barker GL, Edwards KJ, Day IN, Gaunt TR: Predicting the functional, molecular, and phenotypic consequences of amino acid substitutions using hidden Markov models. Hum Mutat 2013;34:57-65.

13 Frousios K, Iliopoulos CS, Schlitt T, Simpson MA: Predicting the functional consequences of nonsynonymous DNA sequence variants-evaluation of bioinformatics tools and development of a consensus strategy. Genomics 2013;102:223-228.

14 Verkerk AO, Remme CA: Zebrafish: a novel research tool for cardiac (patho)electrophysiology and ion channel disorders. Front Physiol 2012;3:255. 